\title{
Coronavirus disease 2019 (COVID-19) vaccination in systemic lupus erythematosus and anti-neutrophil cytoplasmic antibody-associated vasculitis
}

\author{
Jackie $\operatorname{Sim}^{1} \cdot$ Cynthia Ciwei Lim²
}

Received: 18 April 2021 / Revised: 20 April 2021 / Accepted: 22 April 2021 / Published online: 29 April 2021

(c) International League of Associations for Rheumatology (ILAR) 2021

We refer to the article "A practical approach for vaccinations including COVID-19 in autoimmune/autoinflammatory rheumatic disease: a non-systematic review" recently published in this journal [1]. The reasoned and practical suggestions are useful in guiding physicians in their recommendations to their patients with autoimmune diseases. Our prior data showed that systemic lupus erythematosus (SLE) and ANCA-associated vasculitis (AAV) were some of the most frequent biopsy-proven glomerulonephritides to require potent immunosuppressive therapy [2], while immunosuppressants predisposed to increased risk of infections [3]. In light of the raging COVID-19 global pandemic and that COVID-19 infection is associated with significant mortality in chronic diseases, including immunosuppression and kidney disease $[4,5]$, we agree that vaccination against COVID-19 should be advocated for these autoimmune conditions to protect against severe infection [6]. However, early studies of the general population have noted variable acceptance or intention to vaccinate [7]. While such data among those with SLE, lupus nephritis, and AAV is scarce, it is troubling that coverage of even established vaccinations such as those for influenza and pneumococcus remained low among these patients primarily due to concerns about autoimmune disease flares and adverse events [8,9]. Such barriers may persist and prevent uptake of the COVID-19 vaccination among patients with SLE and AAV. Other authors have similarly highlighted that past experience with other vaccines in identifying individual-level determinants of vaccine hesitancy (known as the $5 \mathrm{C}$ model of drivers of vaccine hesitancy, namely confidence, complacency, convenience or

Cynthia Ciwei Lim

cynthia.lim.c.w@singhealth.com.sg

1 Yong Loo Lin School of Medicine, National University of Singapore, Singapore, Singapore

2 Department of Renal Medicine, Singapore General Hospital, Singapore, Singapore constraints, calculation of risk, and collective responsibility) can help focus research into COVID-19 vaccine acceptance or hesitancy and tailor strategies to address their concerns [10]. In the meantime, we eagerly anticipate more information regarding the effectiveness and safety of COVID19 vaccination in individuals with SLE and AAV on various immunosuppressive therapies to allow physicians and patients alike to make informed decisions about COVID-19 vaccination.

\section{Declarations}

Disclosures None.

\section{References}

1. Soy M, Keser G, Atagunduz P, et al (2021) A practical approach for vaccinations including COVID-19 in autoimmune/autoinflammatory rheumatic diseases: a non-systematic review. Clin Rheumatol. https:// doi.org/10.1007/s10067-021-05700-z

2. Lim CC, Tung YT, et al (2018) Epidemiology and risk factors for cytomegalovirus infection in glomerular diseases treated with immunosuppressive therapy. Nephrology 676-681

3. Lim CC, Liu PY, Tan HZ et al (2017) Severe infections in patients with lupus nephritis treated with immunosuppressants: a retrospective cohort study. Nephrology 22:478-484

4. Cheng Y, Luo R, Wang K, et al (2020) Kidney disease is associated with in-hospital death of patients with COVID-19. Kidney Int 829-838

5. Zhou F, Yu T, Du R, et al (2020) Clinical course and risk factors for mortality of adult inpatients with COVID-19 in Wuhan, China: a retrospective cohort study. Lancet 1054-1062 
6. Curtis JR, Johnson SR, Anthony DD, et al (2021) American college of rheumatology guidance for COVID-19 vaccination in patients with rheumatic and musculoskeletal diseases-version 1 . Arthritis Rheumatol. https://doi.org/10.1002/art.41734

7. Sallam M (2021) COVID-19 vaccine hesitancy worldwide: a concise systematic review of vaccine acceptance rates. Vaccines 9:160

8. Chehab G, Richter JG, Brinks R, Fischer-Betz R, Winkler-Rohlfing B, Schneider M (2018) Vaccination coverage in systemic lupus erythematosus-a cross-sectional analysis of the German longterm study (LuLa cohort). Rheumatology 57:1439-1447

9. Loubet P, Kernéis S, Groh M et al (2015) Attitude, knowledge and factors associated with influenza and pneumococcal vaccine uptake in a large cohort of patients with secondary immune deficiency. Vaccine 33:3703-3708

10. Wiysonge CS, Ndwandwe D, Ryan J, et al (2021) Vaccine hesitancy in the era of COVID-19: could lessons from the past help in divining the future? Hum Vaccin Immunother. https://doi.org/ $10.1080 / 21645515.2021 .1893062$

Publisher's note Springer Nature remains neutral with regard to jurisdictional claims in published maps and institutional affiliations. 